\section{Ameliorating the emergency department workflow by involving the observation unit: effects on crowding}

\author{
Primiano lannone \\ Emergency Department, Lavagna \\ Hospital, Italy
}

\begin{abstract}
Crowding adversely affects the performance of emergency departments (EDs) by worsening efficiency, timeliness of care, clinical outcomes and patients' satisfaction. We describe in this study our attempt at improving crowding by modifying the roles and workflow of the ED physicians. The observation unit physician was given the additional duty of prioritizing admissions and managing unclear, complex cases, which were previously under the responsibility of front line emergency physicians. We analyzed two corresponding periods, both before the intervention (9897 ED attendances) in 2012 and after the intervention (10,297 attendances) in 2013. Most of the crowding indices improved significantly, including timeliness of triage, of first medical contact, access to resus area, and overall length of stay in ED. Also, emergency hospital admissions, average specialist consultations and imaging studies per patient decreased significantly. The observation unit workload increased. There was no significant excess of adverse events.
\end{abstract}

\section{Introduction}

Crowding is a reason of great concern for many if not all emergency departments (EDs), and it has been linked to lower quality of care, patients' dissatisfaction and adverse outcomes. ${ }^{1}$ It is well recognized that the input and output components of crowding ${ }^{2}$ are the main determinants of this phenomenon, but they cannot be effectively controlled from the ED standpoint. Although less effective, optimizing the process of care within the ED (i.e. the throughput) is therefore considered the most amenable measure to mitigate crowding. Several of such throughput interventions, including ED workflow reorganization, ${ }^{3}$ have been proposed, but the evidence surrounding their effectiveness is poor. ${ }^{4}$ Also, our ED suffered of crowding in spite of some conventional measures we had adopted (fast track pathways, triage liaison physician, observation unit). Therefore, we analyzed and reshaped the workflow, modifying the roles of ED front line and observation unit physicians. The impact of this quality improvement initiative was measured against acknowledged indicators of crowding and other key ED performance and outcome parameters.

\section{Materials and Methods}

Our ED has a census of 48,000 adult attendances/year and is located within a 392 acute beds non teaching hospital serving a population of 150,000 of Northwestern Italy. We adopt the standard Italian four level triage system. A triage liaison physician operates from 11.00 a.m. to 6 p.m. on weekdays. We have also fast track-pathways for ST elevated myocardial infarction, stroke and abdominal aortic aneurysms. Selected orthopedic, pediatric, ophthalmological, obstetric and gynecological presentations are triaged outside the main ED area. An observation unit within the ED with 6 unmonitored beds for patients with limited care needs amenable to discharge within $24 \mathrm{~h}$ is led by ED physicians (one dedicated physician from 8 a.m. to 8 p.m.).

A focus group of ED physicians and nurses identified the throughput factors of crowding: i) persistent burden of complex, unclear or boarded patients on the front line emergency physicians limiting their ability to assess new attendances timely; ii) competition rather than cooperation for admitting patients; iii) lack of prioritization of admissions; iv) multiple handover and communication failures among ED physicians; v) underutilization of short observation unit.

A workflow delineating new roles of front line and observation unit ED physicians was produced and discussed with ED medical and nursing staff. The new model left the front line physicians in charge of all new attendances and the management of most critical patients. Furthermore, referral by ED front line physicians to the short observation unit physician of all of the patients suitable for observation, boarded patients, or unclear/complex cases needing further assessment or treatment was introduced. The short observation unit physician ultimately decided whether to admit these cases to the short observation unit or manage them in the main ED area. He had also the (new) role of prioritizing all of the emergency admissions except of the most critical cases. No other interventions were instituted at the same time, in particular to triage and referral pathways to specialists. The new organization was fully operative in June 2012.

The following EDWIN ${ }^{5}$ and NEDOCS 6 indicators of crowding were compared using the
Correspondence: Primiano Iannone, Emergency Department, Lavagna Hospital, via don Bobbio 25,16033 Lavagna (GE), Italy.

Tel: +39.018.5329543 - Fax +39.018.5329762.

E-mail: p.iannone@live.com

Key words: Emergency department; Workflow; Crowding; Observation unit.

Conflict of interest: the author declares no potential conflict of interest.

Acknowledgments: special thanks go to Drs. Monica Minardi and Jonathan Coates (critical review), Giuseppina Fera (workflow analysis), Stefania Moretti and Paola Truglio (data control and organizational steering), Matteo Cavallero and Federica Kessisoglu (data extraction), Irene Emiliani (critical review and editorial assistance).

Received for publication: 14 January 2015. Revision received: 15 February 2015.

Accepted for publication: 19 February 2015

This work is licensed under a Creative Commons Attribution 3.0 License (by-nc 3.0).

(C) Copyright P. Iannone, 2015

Licensee PAGEPress, Italy

Emergency Care Journal 2015; 11:4957

doi:10.4081/ecj.2015.4957

electronic ED patients' record archive: i) time from ED arrival (clerk registration) to triage completion/first medical contact/resus area (for critical cases)/disposition (discharge, admission to acute hospital bed or short observation unit); ii) patients who left ED without being seen by a physician.Furthermore, we considered: i) unplanned ED re-attendances within 7 days from ED discharge ${ }^{7}$ ii) admissions to the observation unit; iii) length of stay in the observation unit (observation time); iv) hospital admissions (either from main ED area or observation unit); v) hospital mortality (all causes) within $24 \mathrm{~h}$ from ED arrival (including deaths occurring in the ED); vi) sum of specialist consultations and imaging studies per patient.

The periods from $1^{\text {st }}$ January to $30^{\text {th }}$ April of the two consecutive years 2012 (before) and 2013 (after) were analyzed. Patients triaged to specialist consultants were excluded from this study. Differences of number of events per 1000 cases with their relative $95 \%$ confidence intervals (CIs) were calculated. Differences between unpaired continuous variables were assessed with Student's t-test. Direct methods of standardization were used to adjust for the excess of ED attendances in 2013 using the 2012 period as reference, and standardized differences were reported unless stated otherwise (crude). 


\section{Results}

After excluding the patients triaged outside the main ED area from $1^{\text {st }}$ January to $30^{\text {th }}$ April 2012, there were $9897 \mathrm{ED}$ attendances and $10,297 \mathrm{ED}$ attendances in the corresponding period of 2013. The case mix was well matched for age, sex and higher acuity codes.

Apart from the left without being seen rate, crowding indices improved substantially (Table 1). Standardizing for the excess of ED attendances in 2013 the cumulative time spent in ED (main ED area and eventually observation unit) by all of the patients was of $3328 \mathrm{~h}$ shorter than in 2012 (Figure 1). Also, in 2013 more patients $(+470$ cases) were sent home directly from the main ED area within $4 \mathrm{~h}$ from arrival (+80/1000 ED attendances; Table 1). In 2013 the number of patients admitted to the observation unit increased significantly: +52 patients/1000 ED attendances (Table 2). As consequence, the cumulative time spent by the patients in the observation unit in 2013 increased of $3836 \mathrm{~h}$ with respect to 2012 . However, this extra time was outweighed by an even larger reduction of time spent in the main ED area (-7164 h; Figure 1). The increased workload of observation unit required a dynamic reallocation (on an as needed basis) of one unit of the nursing staff from the main ED or triage area to the obser-

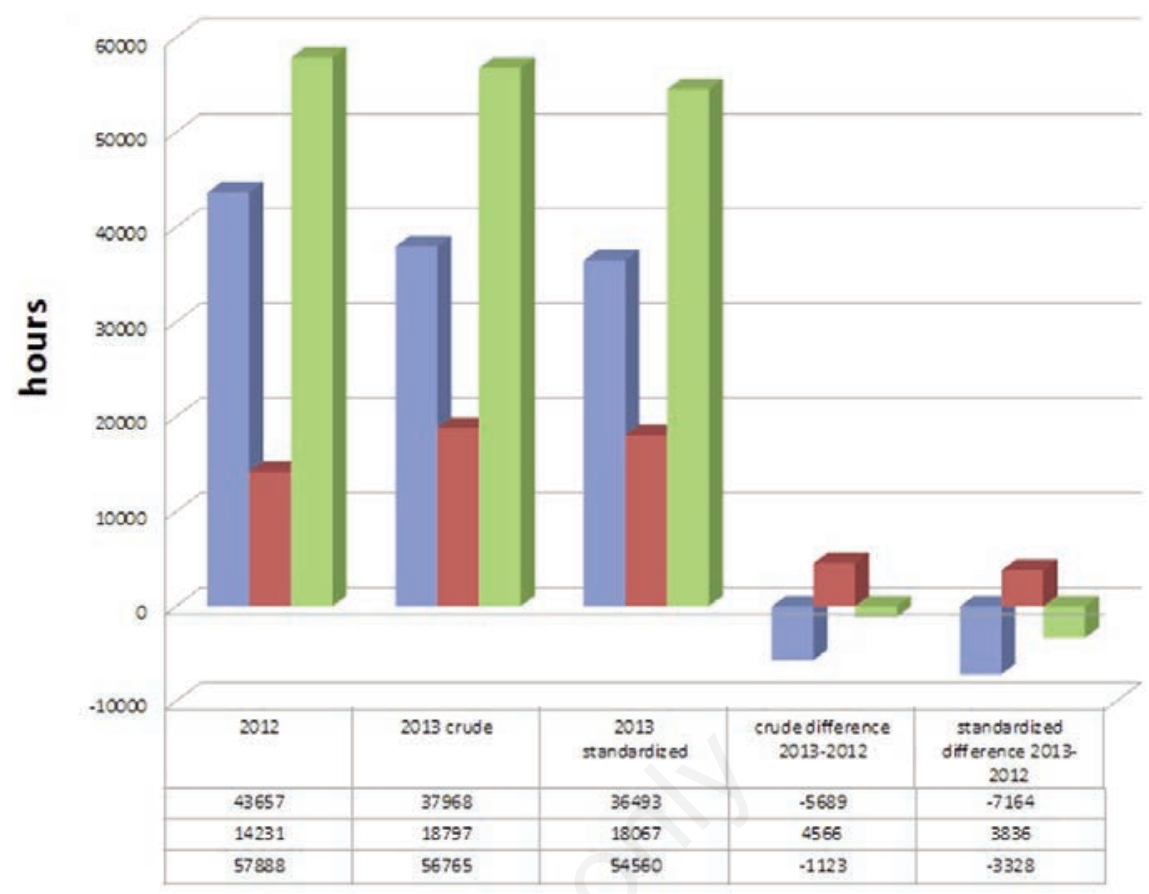

Figure 1. Cumulative length of stay of all the patients in the main emergency department area (blue), in the observation unit (red), and the sum of the two (green) in the periods considered. Standardized values for 2013 are calculated using 2012 as reference (direct standardization) as follows: standardized 2013 value $=$ crude 2013 value* (emergency department 2012 attendances/emergency department 2013 attendances).

Table 1. Key indicators of crowding.

\begin{tabular}{|c|c|c|c|}
\hline Indicator & 2012 & 2013 & $\begin{array}{l}\text { Absolute differences } \\
\times 1000 \text { cases }(95 \% \mathrm{CI})\end{array}$ \\
\hline Patients not triaged within 15 min from ED arrival & $1768(18.6)$ & $1434(14.2)$ & -44 (from -33 to -54 ) \\
\hline Patients waiting $>1 \mathrm{~h}$ from ED arrival to first medical contact & $3524(37.0)$ & $3237(32.0)$ & -50 (from -37 to -63 ) \\
\hline Patients waiting $>30$ min to resus/total resus patients & $764 / 3602(21.2)$ & $438 / 3605(12.1)$ & -91 (from -74 to -107 ) \\
\hline Overall length of stay> $4 \mathrm{~h}^{\circ}$ & $4523(43.9)$ & $3948(39.0)$ & -49 (from -34 to -62 ) \\
\hline Admitted with length of stay $>8 \mathrm{~h} /$ total admitted $^{\circ}$ & 183/1975 (9.3) & $96 / 1637(5.0)$ & -43 (from -59 to -27 ) \\
\hline Discharged with length of stay $>4 \mathrm{~h} / \mathrm{total}$ discharged at home ${ }^{\circ}$ & $2756 / 6866(40.2)$ & $2262 / 7027(32.2)$ & -80 (from -95 to -64$)$ \\
\hline Left without being seen & $424(4.5)$ & $395(3.9)$ & -6 (from -11 to 0$)$ \\
\hline
\end{tabular}

$\mathrm{CI}$, confidence interval; ED, emergency department. ${ }^{\circ}$ Patients admitted to the short observation unit were excluded.

Table 2. Other key outcome indicators.

\begin{tabular}{|c|c|c|c|}
\hline Indicator & $2012^{\mathrm{N}^{\circ}}$ & 2013 & $\begin{array}{l}\text { Absolute differences } \\
\times 1000 \text { cases }(95 \% \text { CI })\end{array}$ \\
\hline Unplanned re-attendances at 7 days & 258/7556 (3.3) & 286/8052 (3.4) & $+1($ from -4 to +7$)$ \\
\hline Admissions directly from ED to acute hospital beds & $1975(20.0)$ & $1637(15.9)$ & -41 (from -30 to -51 ) \\
\hline Admissions to observation unit & $1040(10.5)$ & $1615(15.7)$ & $+52($ from +43 to +61$)$ \\
\hline Admissions from observation unit to acute hospital beds & $350 / 1040(33.7)$ & $590 / 1615(36.5)$ & $+29($ from -9 to +66$)$ \\
\hline Overall admissions to acute hospital beds & $2325(22.9)$ & $2227(21.1)$ & -19 (from -30 to -7 ) \\
\hline Deaths within $24 \mathrm{~h}$ from ED arrival & $61^{\circ}(0.6)$ & $55^{\#}(0.5)$ & $-0.8($ from -1.1 to +3$)$ \\
\hline
\end{tabular}

$\mathrm{CI}$, confidence interval; $\mathrm{ED}$, emergency department. ${ }^{\circ}$ Including 16 deaths occurring in ED; *including 18 deaths occurring in ED. 
vation unit. Since triage times and crowding in main ED area decreased, it was not a critical issue.

In 2013 standardized hospital admissions from the observation unit increased $(+231$ patients) but fewer patients were admitted to acute beds directly from main ED area (-325 patients). Therefore, a net reduction of overall emergency admissions resulted (-19/1000 ED attendances; Table 2). A considerable drop in the average number of consultations and imaging studies per patient was also registered (from $1.15 \pm 1.39$ to $1.05 \pm 1.35$, means \pm standard deviation, $\mathrm{P}<0.05)$. Neither adverse events nor unexpected re-attendances to ED after discharge increased substantially after this intervention (Table 2).

\section{Discussion}

Emergency department crowding is probably the leading problem in the specialty but high quality studies addressing it are still lacking ${ }^{8}$ due to difficulties in reporting complex interventions. ${ }^{9}$ At the ED studied we identified a serious imbalance of physicians' workflow, potentially explaining at least part of the crowding resistant to all of the measures we had adopted previously. Therefore, we conceived a new organization by reassigning the responsibility of complex patients requiring prolonged assessment and or treatment from the front line emergency physicians to the observation unit physician. In this way timeliness of front line emergency care improved considerably, including time to complete triage, of first medical assessment (notably also of the most critical cases) and ED length of stay. However, the number of patients admitted to the observation unit in 2013 increased substantially, raising the doubt that the decrease of the length of stay was fictitious. For example, in 2013 the proportion of ED hospital admissions from the observation unit increased (from 15 to 26\%). This fact contributed undoubtedly to reduce the length of stay in the main ED area of patients waiting for a hospital bed. Instead, the number of patients discharged directly from the main ED area in 2013 within the four hour target increased in absolute terms ( +470 cases), irrespective of the proportion of patients admitted to the observation unit. Furthermore, in 2013 the four months' cumulative length of stay of all the patients (in main ED area and observation unit) decreased of more than 3300 h. Therefore, with the new workflow approximately $10,000 \mathrm{~h}$ of length of stay would have been spared on a yearly basis. Even considering patients held in the observation unit as integral part of the crowding problem of the ED (and not a separate subset of cases usually not taken into account by acknowledged crowding indexes) we obtained a substantial and undisputable benefit from this quality improvement initiative since the overall ED length of stay was reduced in absolute terms, not split between different areas of ED, neither obtained at expense of higher hospital admission rates.

\section{Conclusions}

Rather than a sump phenomenon, the increased number of patients managed by the observation unit physician could reflect a more effective, timely and appropriate processes of care within the ED than it was possible before. The overall reduction of emergency admissions (thanks to a better prioritization and filtering of complex or doubtful cases through the observation unit physician reassessment), as well as of the number of consultations and imaging studies per patient together with the absence of any significant increase of adverse events is consistent with this hypothesis. However, the potential flaws of this model of care need attention. In fact, while allowing more timeliness, and perhaps more appropriate use of resources and sound clinical decisions, this enhanced role of the observation unit physician could lead to an uncontrolled dumping of patients onto the observation area, especially if the cooperation among ED physicians is unsatisfactory or the new decision making and prioritizing tasks are not acknowledged and played adequately. Furthermore, while in our specific setting the observation unit physician was the most suited to do so, others could have the same role elsewhere, depending on ED census, case mix of patients and type of hospital. Noticeably, the analysis of the causes of crowding could differ from one ED to another and legitimately generate solutions quite different from those we have found.

\section{References}

1. Sun BC, Hsia RY, Weiss RE, et al. Effect of emergency department crowding on outcomes of admitted patients. Ann Emerg Med 2013;61:605-11.

2 Asplin BR, Magid DJ, Solberg LI. A conceptual model of emergency department crowding. Ann Emerg Med 2003;42:173-80.

3. King DL, Ben-Tovim DI and Bassham J. Redesigning emergency department patient flows: application of lean thinking to health care. Emerg Med Australas 2006;18:391-7.

4. Hoot NR, Aronsky DA. Systematic review of emergency department crowding: causes, effects and solutions. Ann Emerg Med 2008;52:126-36.

5. Bernstein SL, Verghese V, Leung W, et al. Development and validation of a new index to measure emergency department crowding. Acad Emerg Med 2003;10:93842.

6. Weiss SJ, Derlet $\mathrm{R}$, Ardahl $\mathrm{J}$, et al. Estimating the degree of emergency department overcrowding in academic medical centers: results of the national ED overcrowding study. Acad Emerg Med 2004;11:38-50.

7. Department of Health. A\&E clinical quality indicators. Availabe from: www.wales.nhs.uk/documents/dh_127780 1.pdf

8. Morris ZS, Boyle A, Beniuk $\mathrm{K}$, et al. Emergency department crowding: toward an agenda for evidence based intervention. Emerg Med J 2012;29:460-6.

9. Shepperd S, Lewin S, Straus S, et al. Can we systematically review studies that evaluate complex interventions ? PLoS Med 6:e1000086. 